\title{
Radiométrie visible et infra-rouge du manteau neigeux
}

\author{
J.P. Dedieu, LAMA, Unité de Recherche 344, C.N.R.S.
}

\section{Contexte de la recherche}

L'objectif des campagnes de mesures menées par le Laboratoire de la montagne alpine (LAMA) sur le site expérimental instrumenté au col du lac Blanc $(2800 \mathrm{~m})$ est de pouvoir établir une relation explicite dans des conditions naturelles entre la radiométrie de la neige et les caractéristiques de surface du manteau neigeux : type de grains, densité, teneur en eau liquide (T.E.L.).

Cette relation est tout à fait primordiale pour l'interprétation des images Télédétection transmises par les satellites d'observation de la terre (SPOT, Landsat,...), où les phénomènes sont étudiés à l'échelle du massif alpin.

Dans cette finalité, un prototype de réflectancemètre de terrain a été testé afin de valider les paramètres radiométriques du manteau en surface avec des échantillons collectés in-situ, puis analysés en temps différé dans la chambre froide du C.E.N. (Centre Etudes de la Neige - Météo France). Les échantillons sont prélevés à la fois dans les secteurs de départ et dans les zones d'accumulation après transport de la neige par le vent. Les grains sont alors placés dans un liquide mouillant (iso-octane) afin de stopper la métamorphose en cours et redescendus du site dans un conteneur spécial réfrigéré par neige carbonique.

Cette recherche est menée également avec l'appui logistique du Laboratoire CNRS de Glaciologie et de Géophysique de l'Environnement à Grenoble (L.G.G.E.).

\section{Propriétés spectrales de la neige}

\subsection{Introduction}

Chaque année, environ $30 \%$ de terres émergées de notre planète sont recouvertes de neige. D'où l'importance des études par Télédétection du manteau neigeux dans deux domaines :

- pour des objectifs climatiques (albedo);

- pour des applications hydrologiques (réserves en eau).

L'information de surface obtenue par Télédétection visible est utilisée pour des applications cartographiques à l'échelle d'un massif ou d'un bassin versant et est introduite dans des modèles de prévision de l'écoulement (HALL et Martinec, 1985).

Des informations permettant de distinguer les neiges sèches des neiges humides sont obtenues grâce aux capteurs exploitant le domaine des hyperfréquences passi- ves ou actives. De même, ces longeurs d'onde permettent de restituer une information sur le volume et les hauteurs de neige (RotT, 1990).

\subsection{Spectre de réflectance :}

- Le spectre de réflectance de la neige est dû à la diffusion et à l'absorption de la lumière incidente par les cristaux de glace (diffusion de MIE). La neige est constituée de cristaux de glace dont les dimensions dépendent de son état; le rayon de courbure variant pour exemple entre $50 \mu \mathrm{m}$ (neige fraîche sèche) et $1 \mathrm{~mm}$ (neige de printemps à forte teneur en eau liquide).

- Les nuages, la neige et la glace ont presque le même indice de réfraction et le même coefficient d'extinction de la lumière dans le visible. Par contre, entre 1,55 et 1,75 $\mu \mathrm{m}$, la glace est légèrement plus absorbante que la neige au contraire des nuages qui présentent toujours un indice élevé de réfraction. C'est ce que montre la figure 1. Cette propriété permet de distinguer sur les images neige, glace et nuages, permettant ainsi de lever les ambigultés d'interprétation présentes dans le visible ou le proche infrarouge.

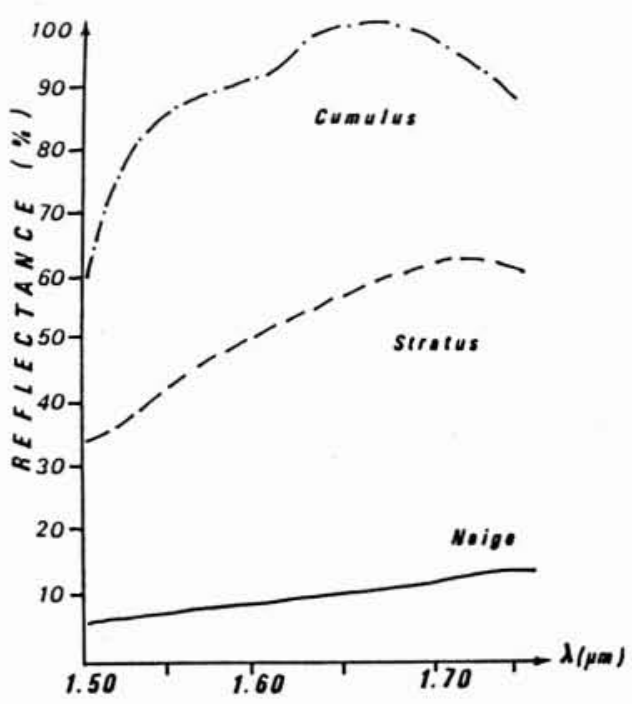

1. Signature spectrale moyen infra-rouge de la neige et des nuages (Valorcin - 1978). 
- D'autre part, si la réflectance de la neige à ses divers états de surface est globalement assez élevée dans le visible, cette valeur diminue dans le proche infra-rouge où la discrimination entre neige fraîche et neige de fusion, est plus aisée. Mais le domaine spectral du Moyen Infra-Rouge est réellement privilégié pour différencier les principaux types de neige, fonction de la taille des cristaux. Cette propriété est représentée par la figure 2 .

\subsection{Influence des facteurs externes}

Les signatures spectrales visible et infra-rouge de la neige ne sont pas celles d'une surface Lambertienne. La luminance d'un tel diffuseur sera dépendante de l'angle de visée et des angles zénithaux du Soleil. Cette propriété est importante pour les études de suivi multi-temporel d'un couvert neigeux à l'échelle d'un bassin versant en période hivernale d'accumulation, puis lors de la fusion printannière.

En zone de montagne, outre les déformations de la géométrie des images dues au relief, les perturbations du signal induites par la présence des ombres portées des versants sont à prendre en compte ; d'où la nécessité d'utiliser des modèles de correction radiométrique des effets dus au relief.

En zones de montagne et de plaine, la signature spectrale des couverts neigeux sera également influencée par la présence ou l'absence d'aérosols. Un modèle atmosphérique sera alors nécessaire.

\section{Méthodologie}

* Collecter des valeurs de réflectance du manteau neigeux au site du col du Lac Blanc pour le rayonnement visible et infra-rouge ;

* comparer ces valeurs avec les caractéristiques de surface du manteau et tout particulièrement avec les échantillons analysés en chambre froide au CEN ;

* confronter les résultats obtenus avec les modèles proposés par certains auteurs (DozIER, 1989) et les réflectances de la neige sur les images fournies par les satellites.

\subsection{Le radiomètre de terrain}

Il s'agit d'un prototype expérimental mis au point par le Laboratoire d'optique atmosphérique de Lille (L.O.A.), version préliminaire du modèle commercialisé en 1991. Ce réflectancemètre se compose de trois éléments séparés (figure 3) :

- une centrale de mesure qui peut être utilisée, soit connectée à un micro-ordinateur, soit de manière autonome puisqu'elle contient une mémoire permanente;

- quatre têtes de collecte du flux hémisphérique descendant dont les bandes passantes sont les suivantes:

$557 \mathrm{~nm}(\Delta \lambda=70)$ (vert)

$638 \mathrm{~nm}(\Delta \lambda=30)$ (rouge)

$850 \mathrm{~nm}(\Delta \lambda=80)$ (Proche infrarouge : PIR)

$1650 \mathrm{~nm}(\Delta \lambda=100)$ (Moyen infrarouge : MIR);

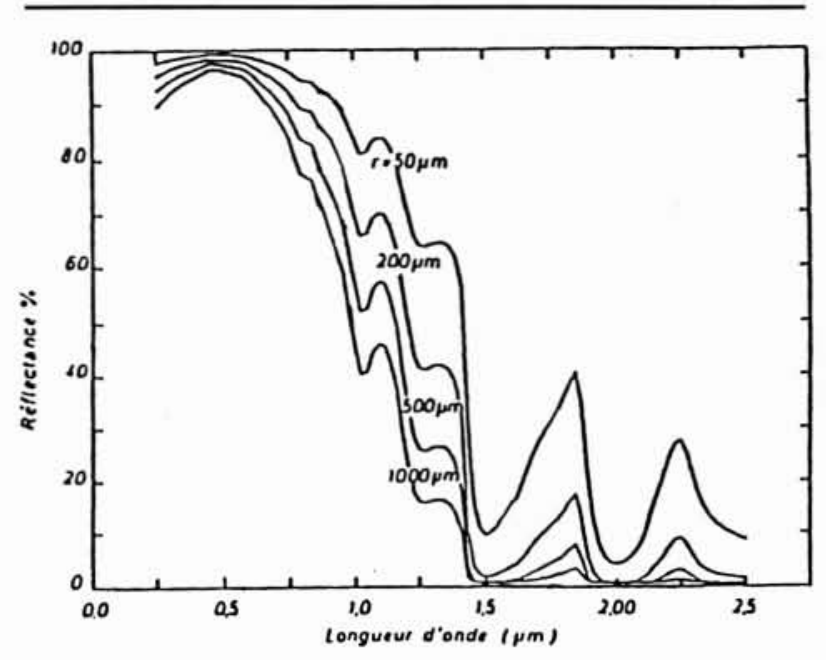

2. Réflectance de la neige pour 4 tailles de cristaux et pour un angle zénithal du soleil de $60^{\circ}$ (Warren - 1981 et Dozier - 1988).

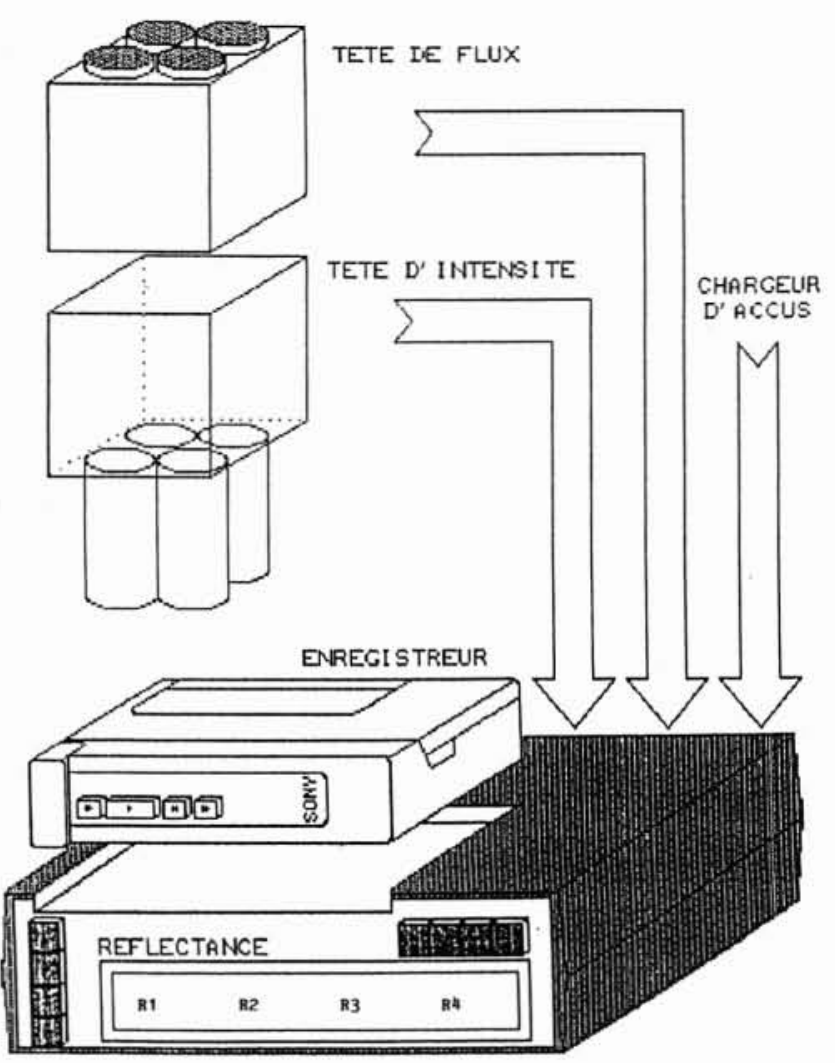

3. Le radiomètre de terrain modèle L.O.A.

- quatre têtes de collecte du rayonnement remontant dans un champ de largeur totale de $15^{\circ}$ et travaillant dans les mêmes bandes.

Chaque capteur avait été étalonné au préalable à l'Institut national de recherche agronomique (INRA), au Groupement d'Avignon qui possède un banc d'étalonnage pour radiomètres de terrain. Les surfaces utilisées ont été le $\mathrm{BaSO}_{4}$ et le HALON. Sur le terrain, avant chaque mesure, une surface de référence est en outre intégrée dans le protocole. 
Il s'agit d'un panneau de peinture dont les caractéristiques de réflectance sont données à la figure 4 .

Pour chaque saisie de mesures sur divers états de surface du manteau, les huit capteurs effectuent 6 enregistrements par minute. Chaque valeur de Réflectance affichée par le radiomètre représente la moyenne du canal considéré sur une durée de 10 secondes.

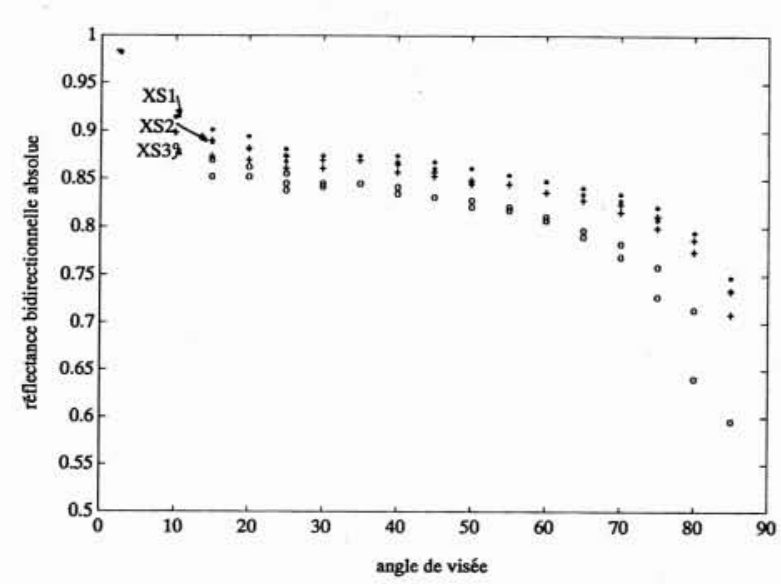

4. Caractéristiques directionnelles du panneau de peinture "zinc " de référence terrain (source: INRA 1990).

\subsection{Expérimentation de terrain}

Les surfaces sur lesquelles les mesures ont été faites présentent une légère pente de 1 à $2^{\circ}$ vers le Nord-Nord Est. Les sites sont soit en exposition Nord (neige fraîche, particules reconnaissables, grains fins), soit en exposition Sud (grains fins, grains ronds) ; un grand nombre de situations nivométéorologiques différentes permettant de saisir tout au long de la saison des caractéristiques de surface fort différentes.

Les capteurs sont placés horizontalement (à l'aide d'un niveau à bulles) à $2 \mathrm{~m}$ de la surface de neige et à une dizaine de mètres l'un de l'autre afin d'éviter au maximum les effets de masque des têtes hémisphériques de flux descendant. La figure 5 expose le principe de montage du radiomètre sur le terrain.

Plusieurs mesures de flux réfléchi n'ont pas seulement concerné l'interface neige/air, mais aussi des strates inférieures du manteau dégagées et lissées avec précaution sur une surface de 1 mètre carré. Les mesures de radiométrie de ces strates sont ensuite reliées au contexte nivométéorologique de constitution et d'évolution de la couche (transport ou non).

Pour chaque mesure de radiométrie, les paramètres suivants sont consignés :

- mesure visuelle à la loupe du type de grain et prise d'échantillon pour mesure ultérieure microscopique en chambre froide (type de cristaux et rayon de courbure); — densité, T.E.L. et épaisseur de la strate étudiée ;

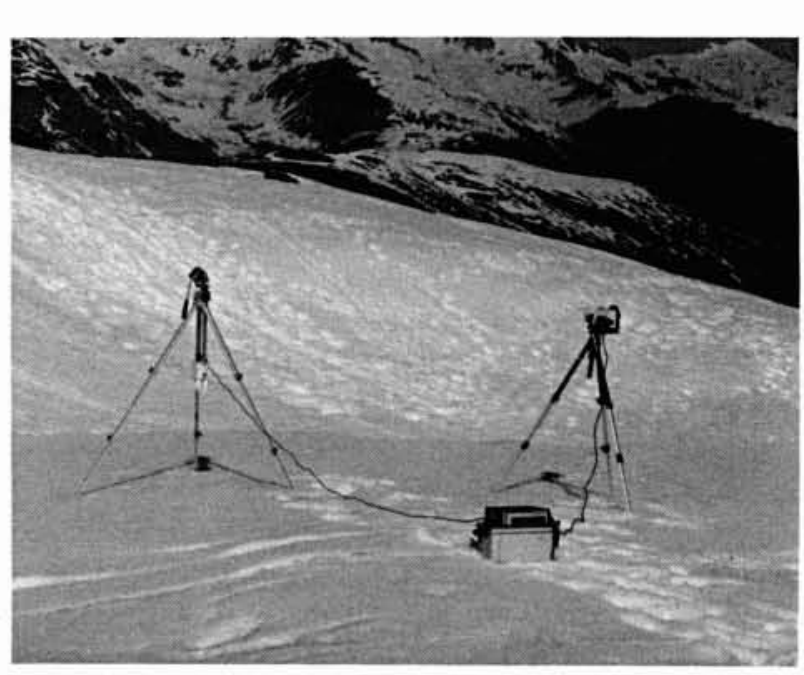

5. Principe de montage du réflectancemètre (deux têtes de flux et une centrale de mesures).

- température de l'air et degré de nébulosité (les mesures présentant au moins $4 / 8^{\mathrm{e}}$ de cirrus ou cumulus devenant difficilement exploitables en raison de très rapides variations de la réflectance Moyen Infra-Rouge);

- jour, heure et altitude (calcul de l'azimut solaire incidant sur les mesures de radiométrie).

\subsection{Mesure de la taille des grains}

Les tailles des grains ont été évaluées pour quasiment tous les échantillons de trois façons différentes :

- in situ, en étalant un peu de neige sur la grille fournie par le CEN on peut évaluer grossièrement le rayon (noté $\mathrm{r}_{\text {visuel }}$ );

- au CEN, en chambre froide, en étalant la neige sur une plaque de verre sous laquelle on a placé une grille graduée. Par macrophotographies enregistrées sur bande vidéo, on peut donner une évaluation visuelle de la taille des grains (paramètre noté $\mathrm{r}_{\text {vidéo }}$ );

- les mêmes macrophotographies (sans grille millimétrée sont utilisées par un programme de traitement d'images qui détermine à partir d'images digitalisées le rayon convexe moyen du grain de neige (paramètre noté $r_{\text {cvx }}$ ).

\section{Résultats}

\subsection{Méthode de calcul de la réflectance}

Pour chacun des quatres canaux du radiomètre, le calcul de la réflectance $\mathbf{R}$ est obtenu par la formule suivante :

$$
R=\frac{\left(V_{r}+Z_{r}\right)}{k \cdot\left(V_{d}+Z_{d}\right)} .
$$


Avec pour chaque longueur d'onde considérée :

$V_{r}=$ signal remontant (luminance).

$V_{d}=$ signal descendant (irradiance).

$Z_{r}=$ tension d'obscurité du canal du flux remontant.

$Z_{d}=$ tension d'obscurité du canal du flux descendant.

$k=$ coefficient d'étalonnage par rapport à une surface de référence recouverte de $\mathrm{BaSO}_{4}$ pour la longueur d'onde considérée.

Les valeurs de $\mathrm{k}$ fournies par l'INRA sont les suivantes :

$k_{\mathrm{MIR}}=1,1507$.

$k_{\mathrm{PIR}}=0,8103$.

$k_{\text {rouge }}=0,5386$.

$k_{\text {vert }}=0,6252$.

Les valeurs des réflectances relatives du $\mathrm{BaSO}_{4}$ obtenues pour chacun des 12 cycles de mesure sont alors moyennées.

Les valeurs de réflectance du $\mathrm{BaSO}_{4}$ utilisées sont les suivantes (source: HAPKE et WELLS, 1981) :

- pour le visible et le proche infrarouge : $R=0,98$;

- pour le canal moyen infrarouge : $R=0,90$.

\subsection{Exemple d'expérimentation sur le terrain}

L'ensemble des mesures collectées sur site étant actuellement en cours de dépouillement, les résultats partiels exposés ci-dessous proviennent d'une campagne de mesures réalisée le 11 avril 1990 en présence du LAMA, du CEN et du LGGE. Ces résultats ont été produits dans un mémoire de fin d'études d'ingénieur au LGGE (Michel FOUQUEMBERGH, 1990) :

Nébulosité: de $4 / 8$ cirrus $(11$ h 30$)$ à $6 / 8$ cumulus (14 h 30).

Echantillon 20 : croûte de regel, gros grains roulés heure $11: 30$.

Mesures :

$R_{\text {vert }}=0,91 \pm 0,02$.

$R_{\text {rouge }}=0,95 \pm 0,02$.

$R_{\mathrm{PIR}}=0,80 \pm 0,07$.

$R_{\mathrm{MIR}}=0,74 \pm 0,08$.

$r_{\text {visuel }}=50 \mu \mathrm{m}$.

$r_{\text {vidéo }}=100-250 \mu \mathrm{m}$.

$r_{\mathrm{CVX}}=230 \mu \mathrm{m}$.

Simulation :

\begin{tabular}{|l|l|l|}
\hline$\Theta=45^{\circ}$ & $\mathrm{r}=50 \mu \mathrm{m}$ & $\mathrm{r}=250 \mu \mathrm{m}$ \\
\hline vert & 0.985 & 0.97 \\
\hline rouge & 0.97 & 0.95 \\
\hline PIR & 0.92 & 0.84 \\
\hline MIR & 0.15 & 0.03 \\
\hline
\end{tabular}

Ici la valeur $r=250 \mu \mathrm{m}$ semble coïncider assez bien pour les canaux rouge et PIR.
Echantillon 18 : neige grains fins et particules reconnaissables heure $12: 00$.

Mesures :

$R_{\text {vert }}=0,87 \pm 0,01$.

$R_{\text {rouge }}=0,92 \pm 0,01$.

$R_{\mathrm{PIR}}=0,74 \pm 0,01$.

$R_{\mathrm{MIR}}=0,50 \pm 0,01$.

$r_{\text {visuel }}=500 \mu \mathrm{m}$.

$r_{\text {vidéo }}=150-300 \mu \mathrm{m}$.

$r_{\mathrm{CVX}}=300 \mu \mathrm{m}$.

Simulation :

\begin{tabular}{|l|l|l|l|}
\hline$\Theta=45^{\circ}$ & $\mathrm{r}=150 \mu \mathrm{m}$ & $\mathrm{r}=500 \mu \mathrm{m}$ & $\mathrm{r}=650 \mu \mathrm{m}$ \\
\hline vert & 0.98 & 0.96 & 0.95 \\
\hline rouge & 0.96 & 0.92 & 0.91 \\
\hline PIR & 0.875 & 0.78 & 0.75 \\
\hline MIR & 0.05 & 0.015 & 0.001 \\
\hline
\end{tabular}

Ici, c'est un rayon plus important que ceux proposés par les mesures visuelles qui semble être le plus approprié si l'on observe les canaux rouge et PIR.

Echantillon 19 : neige grains ronds sous couche heure $12: 30$.

Mesures :

$R_{\text {vert }}=0,93 \pm 0,01$.

$R_{\text {rouge }}=0,94 \pm 0,01$

$R_{\mathrm{PIR}}=0,66 \pm 0,01$.

$R_{\mathrm{MIR}}=0,74 \pm 0,01$.

$r_{\text {visuel }}=50 \mu \mathrm{m}$.

$r_{\text {vidéo }}=50-75 \mu \mathrm{m}$.

$r_{\mathrm{CVX}}=170 \mu \mathrm{m}$.

Simulation:

\begin{tabular}{|l|l|l|}
\hline$\Theta=40^{\circ}$ & $\mathrm{r}=50 \mu \mathrm{m}$ & $\mathrm{r}=170 \mu \mathrm{m}$ \\
\hline vert & 0.98 & 0.975 \\
\hline rouge & 0.97 & 0.95 \\
\hline PIR & 0.91 & 0.86 \\
\hline MIR & 0.14 & 0.05 \\
\hline
\end{tabular}

Ici, la taille de grain donnée par le modèle avoisinerait plutôt $200 \mu \mathrm{m}$ si l'on accepte une erreur d'environ $20 \%$ sur le canal PIR. 
Echantillon 34 : même neige que Ech. 19 après exposition au soleil heure $14: 30$.

Mesures :

$R_{\text {vert }}=0,91 \pm 0,01$.

$R_{\text {rouge }}=0,92 \pm 0,01$

$R_{\mathrm{PIR}}=0,63 \pm 0,01$.

$R_{\mathrm{MIR}}=0,69 \pm 0,01$.

$r_{\text {visuel }}=500 \mu \mathrm{m}$.

$r_{\text {vidéo }}=300-400 \mu \mathrm{m}$.

$r_{\mathrm{CVX}}=230 \mu \mathrm{m}$.

Simulation :

\begin{tabular}{|l|l|l|l|}
\hline$\Theta=36^{\circ}$ & $\mathrm{r}=200 \mu \mathrm{m}$ & $\mathrm{r}=500 \mu \mathrm{m}$ & $\mathrm{r}=800 \mu \mathrm{m}$ \\
\hline vert & 0.97 & 0.953 & 0.94 \\
\hline rouge & 0.95 & 0.92 & 0.895 \\
\hline PIR & 0.85 & 0.76 & 0.71 \\
\hline MIR & 0.04 & 0.011 & 0.005 \\
\hline
\end{tabular}

Ici, la valeur du canal PIR est assez faible laissant augurer une grande taille de grain.

\subsection{Discussion}

Ces résultats partiels permettent de remarquer plusieurs faits :

- la trop forte nébulosité perturbe la mesure dans le canal Moyen Infra-Rouge, qui ne donne guère de réponse en accord avec la modélisation simulée des réflectances. Les mesures réalisées en 1991 sur d'autres types de neige ou bien sur des surfaces identiques en l'absence de couverture nuageuse offrent des résultats bien mieux corrélés avec les modèles. Ces informations sont en cours de dépouillement pour une validation définitive d'un modèle liant Réflectance et Taille de grains en vue d'une interprétation plus pertinente des images satellites infra-rouge à l'échelle d'un massif ;

- comme l'indiquent Wiscombe et WARREN (1981), les canaux du visible sont très sensibles à la contamination par des impuretés. Comme toutes les neiges rencontrées étaient de vieilles neiges puisqu'il n'avait pas neigé récemment, il est normal que les valeurs de réflectance obtenues expérimentalement soient systématiquement plus faibles que les valeurs prévues par le modèle pour une neige pure;

- si l'on compare qualitativement les valeurs de réflectance dans les différentes bandes, on peut constater que l'évolution se fait bien suivant ce que prévoit la théorie, c'est-à-dire que le canal vert prend des valeurs voisines des valeurs du canal rouge et que les canaux PIR et surtout MIR (lorsqu'il fonctionne) prennent des valeurs nettement plus faibles ;

- la taille des grains n'est pas donnée avec une grande précision et on peut constater que les interprétations peuvent rapidement varier du simple au double, suivant la méthode de mesure et l'opérateur. Ce fait illustre bien la difficulté qu'il y a de définir rigoureusement une taille de grain.

Ultérieurement, le programme de recherche devra être étendu aux applications nivologiques des capteurs microondes, en relation avec l'étude photogrammétrique du site expérimental (hauteurs de neige, volume des transports). Par ailleurs, les capteurs microondes sont sensibles à des caractéristiques du manteau neigeux qui sont des paramètres du risque d'avalanche:

humidité, stratification, givre de profondeur.

Des études plus approfondies sont en cours (MÄTZLER, 1987), au moyen de divers types de capteurs (actifs et passifs).

\section{Références}

Dedieu, J.P. et al. 1989. Apport des données SPOT et Landdsat TM pour le suivi de la fusion nivale, Société Française de Photogrammétrie et de Télédétection, Bull. $\mathrm{n}^{\circ} 115$, 49-52.

Dozier, J. 1989. Spectral signature of Alpine Snow Cover from the Landsat Thematic Mapper, Remote Sens. Environ., 28, 9-22.

HALl, D.K. and J. MARTINEC. 1985. Remote sensing of ice and snow, ed., Chapman and Hall, London, 189 pp.
MÄTZLER, C. 1987. Microwave sensors for measuring avalanchecritical snow parameters, Avalanche Formation, Movement and Effects, IAHS Publ. $\mathrm{n}^{\circ} 162$.

Rotr, H. 1990. Snow and land ice in the climate system : research problems and possibilities of remote sensing, Remote sensing and the earth's environment, Proceedinds of Summer school held at Alpbach, Austria, 1989, ESA SP301, 61-75.

WISCOMBE, N.J., WARREN, S.G. 1981. A model for the spectral albeds of snow, Journal of the Atmospheric Sciences, vol. 37, 2712-2745. 